\title{
Angina crónica estable-Guía de manejo clínico en atención primaria Parte I
}

\section{1-Introducción}

La enfermedad cardíaca isquémica debida a la obstrucción de las arterias coronarias por arteriosclerosis es la causa más frecuente de muerte en el mundo occidental, donde representa alrededor del 33\% de la mortalidad total. ${ }^{1}$ La manifestación más común de dicha patología es la angina crónica estable, siendo el "primer aviso clínico" en más de la mitad de los pacientes portadores de cardiopatía isquémica. El desarrollo de una metodología de estudio y tratamiento, tendiente a unificar criterios, adquiere una manifiesta importancia, ya que la mayoría de estos pacientes se presenta en consultorios médicos que habitualmente se hallan alejados de centros hospitalarios de alta complejidad cardiovascular.

La presente guía clínica consta de dos partes, la primera se refiere al diagnostico y valoración del riesgo, y la segunda al tratamiento con especial énfasis en el tratamiento médico. El texto se basa principalmente, aunque no completamente, en la guía de manejo de las Fuerza de Tareas del American College of Cardiology y el American Heart Association (ACC/AHA), publicada simultáneamente en Junio de 1999, en el Journal of the American College of Cardiology y en Circulation. ${ }^{2}$

La evidencia de las recomendaciones de la guía, está agrupada según su forataleza en:

A- La mayor y mejor evidencia. Los datos provienen múltiples estudios clínicos randomizados y controlados.

B- Evidencia intermedia. Los datos provienen de un número limitado de estudios randomizados que incluyen pocos pacientes o cuidadosos análisis de estudios no randomizados observacionales.

C- Menor evidencia que proviene de recomendaciones de consensos de expertos.

La guía combina las opiniones de expertos y el grado de la evidencia en niveles de recomendación I, II y III.

Clase I: Condición para la cual hay evidencia y/o acuerdo general que determinado procedimiento o tratamiento es útil y efectivo.

Clase II: Condición para la cual la evidencia es conflictiva y/o hay divergencia de opiniones sobre la utilidad y eficacia de un procedimiento o tratamiento.

Clase II a: El peso de la evidencia/opinión, es en favor de su utilidad y eficacia.

Clase II b: El peso de la evidencia/opinión esta menos establecido.

Clase III: Condición para la cual hay evidencia y/o acuerdo general que determinado procedimiento o tratamiento no es útil ni efectivo y en algunos casos puede ser perjudicial.

\section{2-Fisiopatología:}

Para tomar determinaciones médicas correctas es necesario conocer la fisiopatología del evento en estudio; sólo de esta manera podremos elaborar conductas diagnósticas y de tratamiento adecuadas.

Cuando la demanda de oxígeno supera la capacidad del transporte hasta el tejido miocárdico, ante cualquier nivel de exigencia, se establece una situación de isquemia. Sea cual fuese el mecanismo desencadenante de la isquemia las consecuencias son similares. Se genera una secuencia de acontecimientos denominado cascada isquémica. Primero el compromiso celular genera una alteración en la función diastólica, luego sistólica con la aparición de áreas de hipoquinesia segmentaria y caída de la fracción de eyección. Esta primera alteración es objetivable mediante el ecocardiograma que detecta la hipoquinesia, y por la perfusión por talio, que muestra áreas con menor captación que desaparecen en el reposo. Si persisten los cambios en la polaridad celular se producen cambios eléctricos, que pueden ser detectados por el electrocardiograma. Por último, si la isquemia sigue persistiendo, aparece la manifestación clínica cardinal: la angina de pecho. ${ }^{3}$ La angina que es crónica y es estable requiere de la combinación de dos condiciones: la presencia de obstrucciones fijas en cualquier nivel de los vasos, que permite la perfusión con adecuada provisión de oxígeno solo en reposo y hasta un cierto nivel de demanda (o consumo), y un aumento del consumo miocárdico de oxígeno provocado por el ejercicio, por un estado emocional o por un apremio en el contexto de una prueba diagnóstica.

La isquemia es secundaria a un aumento de la demanda, y en él la situación isquémica se desencadena solo si el aumento del consumo de oxígeno supera un nivel crítico llamado umbral.

Por lo tanto la crisis isquémica clínica suele aparecer en situaciones que pueden ser reproducibles por un esfuerzo físico con aumento del doble producto (frecuencia cardiaca x presión arterial sistólica). Pero ese umbral isquémico puede modificarse bajo algunas circunstancias. De esta manera nos encontramos con dos clases de angor crónico estable (ACE): 4

1-Angina estable con umbral fijo: Es predecible, presenta escasa tendencia a la oclusión coronaria. Las pruebas provocadoras de espasmo son negativas y fundamentalmente la capacidad funcional clínica se relaciona con el nivel de reserva coronaria. El paciente siempre siente angina ante un esfuerzo similar o parecido.

2-Angina estable con umbral variable: La variabilidad del umbral clínico se halla modulada o regulada en principio por cambios provocados por variaciones de la luz arterial por espasmo de las arterias coronarias sobre una placa previa (componente fijo + componente variable). Estos pacientes experimentan angina con actividad física de distinta intensidad, sin que esto implique empeoramiento. Típicamente estos pacientes refieren buenos días en que son capaces de desarrollar una actividad física vigorosa sin angina, y malos días en que desarrollan angina a un esfuerzo mucho menor.

En la práctica todos los pacientes con ACE tienen en mayor o menor medida un componente variable y es importante distinguirlos porque si este es muy pronunciado el tratamiento puede ser distinto. Ejemplos de componente variable en un paciente con ACE son la angina nocturna, la angina matinal que mejora durante el día, o la angina provocada por emociones.

\section{3-Definición:}

La ACE se define como aquella que no modificó su frecuencia e intensidad en los tres meses previos. Puede estar asociada o no la presencia de infarto de miocardio previo.

Esto debe significar, además, que posee un patrón predecible en frecuencia y factores precipitantes y este patrón debe respetarse en los últimos tres meses. 4

Una vez realizado el diagnóstico de ACE, es muy importante categorizar la clase funcional del dolor anginoso, es decir, la intensidad de la actividad física que desencadena angina. Esto permite valorar la calidad de vida del paciente y evaluar la respuesta terapéutica a cualquiera de los tratamientos instituidos. La clasificación mas utilizada es la de la Sociedad Canadiense de Cardiología.

\section{Angina estable-Clasificación Funcional-SCC}

Clase I: La actividad física habitual no provoca angina. La angina aparece con el trabajo muy intenso o extenuante.

Clase II: Limitación discreta de las actividades normales. Dolor al caminar o subir escaleras apurado, caminar en pendiente, después de comer, en el frío, caminar más de dos cuadras o subir dos pisos de escalera. Clase III: Limitación acentuada de la actividad física. Dolor al caminar una o dos cuadras en plano y subir más de un piso por escaleras en condiciones normales.

Clase IV: Incapacidad de efectuar cualquier actividad sin sentir molestias. Puede haber dolor en reposo.

\section{4- Diagnóstico}

\section{A- CARACTERIZAR EL DOLOR DE PECHO}

El dolor precordial es la manifestación más constante, importante y conocida de la enfermedad coronaria. Constituye un elemento de gran jerarquía tanto en el diagnóstico como en la evaluación de la gravedad de la enfermedad. El diagnóstico de ACE es básicamente clínico, por lo tanto, es importante que el médico conozca sus características. 
Se han estudiado las características del dolor y su relación con la presencia de enfermedad coronaria (EC). Los datos del mismo, junto a los factores de riesgo coronario, nos ayudarán a estimar la probabilidad de su origen coronario. La presencia de un dolor típico prácticamente confirma la presencia de EC. Si el dolor fuese atípico, se necesitan pruebas diagnósticas que acerquen o alejen el diagnóstico. Si el dolor fuese no anginoso podríamos en principio descartar EC. 56

Si se considera que el dolor de pecho puede deberse a angina, el segundo paso es evaluar si existe riesgo de un evento agudo. Esto implica determinar si tuvo dolores en reposo o ante mínimos esfuerzos, si está empeorando progresivamente la clase funcional, o si presentó dolores prolongados. Recomendación Clase I, nivel de evidencia B

\section{Tabla 1: Clasificación del dolor de pecho}

Típico: es aquel de 1) localización retroesternal, 2) Se manifiesta con
el esfuerzo y 3) Cede con el reposo y/o con nitritos (habitualmente en
los siguientes 10 minutos). Para ser considerado como tal debe tener
las tres características.
Atípico: es aquel que presenta dos de las tres características expuestas
previamente.
No anginoso: es aquel que no reúne las características mencionadas o
cumple con una sola de ellas.
Fuente: Diamond GA, Forester JS. N Engl J Med 1979; 300:1350-1358

Tabla 2. Probabilidad previa de EC en pacientes con dolor de pecho según sexo y edad. ${ }^{7}$

\begin{tabular}{|lcccccc|}
\hline & $\begin{array}{c}\text { Dolor de pecho } \\
\text { no anginoso }\end{array}$ & \multicolumn{2}{c|}{ Dolor Atipico } & \multicolumn{2}{c|}{ Dolor Típico } \\
\hline Edad & Hombre & Mujer & Hombre & Mujer & Hombre & Mujer \\
\hline $30-39$ & 4 & 2 & 34 & 12 & 76 & 26 \\
\hline $40-49$ & 13 & 3 & 51 & 22 & 87 & 55 \\
\hline $50-49$ & 27 & 14 & 72 & 51 & 94 & 86 \\
\hline
\end{tabular}

Cada valor representa el porcentaje de EC significativa evaluada por un cateterismo.

B- Laboratorio para descartar condiciones asociadas y evaluar perfil de riesgo-

Se recomienda realizar los siguientes estudios de laboratorios iniciales para evaluar condiciones asociadas y factores de riesgo: Hemoglobina, glucemia y perfil lipídico. Recomendacion clase I, con nivel de evidencia $C$

\section{C- Pruebas no invasivas}

1-Rx de torax: Indicada en pacientes con clínica de insuficiencia cardíaca, valvulopatía, enfermedad pericárdica 0 aórtica. Recomendación Clase I, nivel de evidencia B

\section{2-ECG}

Un ECG debe ser registrado en todo paciente con sospecha de angina de pecho, aunque su papel más importante tiene lugar en el diagnóstico y seguimiento de pacientes inestables. En los pacientes de consultorio, el ECG es normal en cerca del 50\% de los pacientes con ACE. La presencia de ondas $Q_{0}$ o de cambios en el ST-T consisitentes con isquemia favorecen el diagnóstico de angina y ayuda a la estratificación del riesgo. La presencia de hipertrofia ventricular izquierda aumenta la probabilidad que la molestia precordial se deba a angina de pecho. Recomendación Clase I, nivel de evidencia B

\section{3- Pruebas Evocadoras de isquemia}

La decisión de utilizar algunas de las pruebas funcionales para evocar isquemia deben basarse en la probabilidad previa que tiene el paciente de padecer EC. La realización de estas pruebas en pacientes con probabilidad baja de padecer $\mathrm{EC}$, aumenta considerablemente el riesgo de generar resultados falsamente positivos. A su vez, si el paciente tiene una probabilidad previa de EC muy elevada, un resultado negativo no cambiará la sospecha clínica.

El grupo que más se beneficia con las pruebas diagnósticas es aquel que incluye a los pacientes con una probabilidad previa intermedia de padecer enfermedad coronaria, por ejemplo aquellos con dolores atípicos y uno o dos factores de riesgo coronario. En este grupo, el resultado de la prueba (cualquiera sea), tiene una gran influencia en la probabilidad posterior y ayuda al médico a tomar una conducta. ${ }^{9}$

\section{3.a) Recomendaciones de Prueba Ergométrica Graduada (PEG) ${ }^{2}$}

La PEG es una excelente prueba dado su bajo costo y amplia accesibilidad. Su sensibilidad y especificidad promedio son del $65 \%$ y $89 \%$ respectivamente. En términos generales esta debería ser la primera prueba diagnóstica a solicitar en los pacientes en que se sospecha enfermedad coronaria. ${ }^{9}$

La situación ideal para pedir una PEG, es aquel paciente con probabilidad intermedia de EC, que pueda hacer ejercicio, y con un ECG sin signos agudos de isquemia (infradesnivel no mayor a $1 \mathrm{~mm}$.) ni alteraciones que impidan su interpretación (como bloqueo completo de rama izquierda). Recomendación Clase I, nivel de evidencia $\mathbf{B}$.

Los elementos a tener en cuenta de la.PEG son los siguientes:

* Aparición de angor (peor pronóstico cuando el angor es desencadenado por esfuerzos pequeños).

* Infradesnivel del ST. Se considera diagnóstico un infradesnivel de por lo menos un $1 \mathrm{~mm}$ en los hombres y $1,5 \mathrm{~mm}$ en las mujeres en por lo menos dos derivaciones.

* Desarrollo de hipotensión y/o bradicardia en ausencia de disfunción ventricular conocida: estos signos sugieren una enfermedad severa.

* Aparición de extrasístoles ventriculares o taquicardia ventricular durante el ejercicio o la recuperación.

3.b) Recomendaciones de evaluación funcional con imágenes (EFI) en pacientes que pueden hacer ejercicio ${ }^{2}$

La EFI están indicadas en pacientes que tienen alteraciones del ECG en reposo que impiden la interpretación de la PEG. Estas son bloqueo completo de rama izquierda, hipertrofia ventricular izquierda con alteraciones en la repolarización ventricular, mas de $1 \mathrm{~mm}$. de infradesnivel del ST, ritmo de marcapasos. A su vez, la PEG brinda una información limitada en los pacientes que fueron sometidos a una cirugía de revascularización miocárdica (CRM) o angioplastia, debido a alteraciones del ECG basales y la incapacidad para localizar la enfermedad. Si el paciente puede realizar ejercicio, los estudios con imágenes indicados son: Perfusión miocárdica con talio en reposo y esfuerzo, ventriculograma radioisotópico y ecografía-ejercicio. Recomendación Clase I, nivel de evidencia B.

Las imágenes de la perfusión con talio pueden ser planas e interpretadas visualmente (estudio convencional) o tridimensionales e interpretadas con la asistencia de una computadora (SPECT). La prueba convencional tiene una sensibilidad del $84 \%$ y una especificidad del $87 \%$. La sensibilidad es mayor que la de la PEG y asciende al $95 \%$ con el uso del SPECT. En realidad la performance de la prueba depende en parte del centro en el que se realiza y la dosis de radioisótopo utilizado, pudiendo ser semejantes a las de la PEG ECG, aunque su costo es tres a cuatro veces mayor.

El fundamento de las pruebas con ecocardiografia, es la detección de hipomotilidad causada por isquemia. El ECO- ejercicio es operador dependiente tanto en su realización como interpretación ya que depende de conseguir un máximo esfuerzo y una rápida imagen. La sensibilidad de esta prueba es de $74 \%$ al $91 \% \%$ y la especificidad es del $64 \%$ al $93 \%$. Estudios comparativos en centros especializados demuestran mayor sensibilidad que la PEG e igual performance que las pruebas radioisotópicas. 10

3.c) Recomendaciones de Pruebas Funcionales con Imágenes en pacientes que no pueden hacer ejercicio ${ }^{2}$

Muchos pacientes están incapacitados para hacer ejercicio y son incapaces de alcanzar la frecuencia cardíaca adecuada para detectar isquemia. Generalmente se debe a osteoartritis, enfermedad vascular periférica, enfermedad pulmonar, o malas condiciones físicas. En 
estos pacientes se utilizan drogas que producen una apremio, y en forma graduada inducen isquemia. Estas drogas son vasodilatadores como el dipiridamol y adenosina, e inotrópicos como la dobutamina. Las imágenes se visualizan utilizando perfusión miocárdica con radioisotopos o ecocardiografía.

La perfusión miocárdica con dipiridamol o adenosina, o la ecocardiografía con dobutamina, están indicadas en pacientes para evaluación diagnóstica o pronóstica de EC, impedidos de hacer ejercicio. Recomendación Clase I, nivel de evidencia B.

La perfusión miocárdica con dipiridamol o adenosina, o la ecocardiografía con dobutamina está indicada para evaluación de isquemia miocárdica en pacientes impedidos de hacer ejercicio, con un procedimiento de revascularización previo (CRM o angioplastia). Recomendación Clase I, nivel de evidencia B.

El uso del dipiridamol y la adenosina se basa en la propiedad de estos fármacos de producir una importante vasodilatación en las arterias coronarias sanas. Debido a que los vasos afectados por lesiones arteriescleróticas tienen una respuesta menor o nula a estas drogas, su administración produce una alteración en el flujo coronario que ocasiona un fenómeno de "robo" que precipita la isquemia. La perfusión miocárdica con talio y dipiridamol tiene una sensibilidad y especificidad en promedio de $86 \%$ y $71 \%$ respectivamente.

Un talio dipiridamol normal, es de buen pronóstico, inclusive en presencia de EC conocida. Una revisión de 16 estudios que incluían 3594 pacientes, mostró que los pacientes con prueba negativa tenían una tasa de infarto y muerte del $0,9 \%$ anual, similar a la de la población general.

Otro tipo de prueba utilizada es el estudio (principalmente el ecocardiograma) que utiliza dobutamina como prueba de apremio farmacológico. Su sensibilidad oscila entre el 80 y el $97 \%$ y una especificidad cercana al $90 \%$. Los datos a objetivar con este tipo de estudios es la detección de trastornos tranisitorios de la motilidad.

\section{D) Pruebas invasivas}

Recomendaciones de Cinecoronariografia (CCG) para establecer un diagnóstico, en pacientes con sospecha de angina ${ }^{2}$

Dado su alto riesgo tienen indicación de CCG pacientes con angina de pecho conocida o probable, que sobreviven a un episodio de muerte súbita. Recomendación Clase I, nivel de evidencia $\mathbf{B}$.

En algunas circunstancias clínicas, hay pacientes con diagnóstico incierto de angina de pecho luego de pruebas funcionales, en que es preciso tener un diagnóstico; en los que el riesgo de la angina supera el riesgo de hacer una CCG. Recomendación Clase IIa, nivel de evidencia C.

\section{5- Estratificación del riesgo}

Uno de los objetivos más importantes en el manejo de los pacientes coronarios estables, es identificar a aquellos con mayor riesgo de padecer muerte súbita, infarto de miocardio y miocardiopatía isquémica e implementar una adecuada estrategia terapéutica. A esto se denomina estratificación del riesgo.

La valoración correcta del riesgo es un paso fundamental en la evaluación de los pacientes con EC, ya que permite optimizar el beneficio terapéutico según la necesidad de cada paciente. Permite discriminar a aquellos pacientes con un riesgo alto o intermedio y que se beneficiarían con procedimientos de revascularización (angioplastia y cirugía de puente coronario) de aquellos con riesgo bajo o intermedio que no requieren procedimientos invasivos y se benefician con tratamiento médico. Esta valoración aporta una noción del riesgo en un momento dado y en un paciente determinado, sin embargo debe tenerse presente que la EC es una entidad dinámica. Las necesidades de un paciente en un momento pueden ser diferentes en otro. Por esto es importante tener un criterio de seguimiento y control longitudinal.
Los pacientes que probadamente tienen mayor riesgo y se benefician con procedimientos de revascularización son aquellos con compromiso del tronco de la coronaria izquierda, enfermedad de tres vasos con disminución de la función ventricular, y enfermedad de dos vasos que incluya la descendente anterior proximal con disminución de la función ventricular. ${ }^{13}$

La determinación del riesgo del paciente coronario se realiza considerando tres factores interrelacionados:

\section{Extensión de la enfermedad \\ 2.Grado de isquemia \\ 3.Función ventricular}

1. Extensión de las lesiones que comprometen las arterias coronarias: para definirla se considera la cantidad de vasos comprometidos y se nombra de acuerdo al número de arterias comprometidas. El árbol coronario tiene dos arterias: la coronaria derecha (CD) y la coronaria izquierda (CI), que después del tronco se divide en descendente anterior (DA) y circunfleja (CX). De àcuerdo a esto, el compromiso puede ser del tronco de la CI y/o de uno, dos o tres vasos. Cuando el compromiso es del tronco de la CI o existe mayor cantidad de vasos comprometidos, el riesgo es mayor. 12

La extensión de la isquemia y los vasos comprometidos pueden inferirse de acuerdo al compromiso de las derivaciones afectadas en la PEG, y a la cantidad de segmentos afectados en los estudios de imágenes.

2. Grado de isquemia: Una forma de evaluar la misma es la clase funcional de la angina. La segunda forma de evaluarla es mediante el uso de pruebas funcionales, determinando el esfuerzo en que aparecen los síntomas y/o los cambios isquémicos (ya sean electrocardiográficos, en la motilidad objetivados por el ecocardiograma o en la perfusión detectados por cámara gamma).

\section{Criterios de riesgo de las pruebas funcionales}

En la evaluación del riesgo de cualquier prueba funcional, debe tenerse presente que los indicadores de mayor riesgo son aquellos que muestran directa o indirectamente la aparición de disfunción ventricular. La duración del ejercicio por ejemplo es uno de los indicadores de pronostico independiente mas importantes. ${ }^{9}$

a) Criterios de riesgo en la PEG 5,10

Riesgo bajo:

1-Angor y/o infradesnivel del ST horizontal o descendente mayor de 1 $\mathrm{mm}$ a capacidad funcional superior a los 10 mets. (No debe estar acompañado de otros signos de alto riesgo).

2-Infradesnivel del ST de morfología lentamente ascendente entre 1 y 2 $\mathrm{mm}$ a capacidad funcional superior a los 5 mets sin la presencia de angor.

En aquellos pacientes con bajo riesgo ergométrico, se deberá seguir con tratamiento médico completo. Recomendación Clase I.

\section{Riesgo moderado:}

1-Angor y/o infradesnivel del ST horizontal o descendente mayor a 1 $\mathrm{mm}$ a capacidad funcional entre 4 y 10 mets.

2-Arrtimia ventricular (excluyendo taquicardia ventricular) asociada con angor y/o desnivel del ST.

3-Caída de la presión arterial sistólica asociada con evidencias de isquemia a alta capacidad funcional (mayor a 10 mets) luego de haber alcanzado cifras elevadas durante el esfuerzo.

Los pacientes en los cuales la evaluación sea de riesgo moderado, deberán ser avaluados con un estudio funcional por imágenes (EFI). Recomendación Clase I

Riesgo alto:

1-Infradesnivel del segmento ST horizontal o descendente superior a $2 \mathrm{~mm}$ y/o angor típico a baja capacidad funcional (menor a 4 mets).

2-Caída de la presión arterial igual o mayor de $20 \mathrm{mmHg}$ asociada con 
otras variables isquémicas.

3-Arritmias ventriculares complejas a baja capacidad funcional y asociadas con cambios del ST y/o angor.

4-Aparición de los cambios patológicos del ST en derivaciones múltiples. 5-Supradesnivel del ST superior a $1 \mathrm{~mm}$ en derivaciones sin secuela de IAM. 6-Recuperación tardía (más de 6 minutos) de los cambios isquémicos del segmento ST.

Es consenso que los pacientes cuya PEG sea de alto riesgo deban ser sometidos a una cinecoronariografía (CCG). Recomendación Clase I

b) Criterios de riesgo de la perfusión miocárdica

Riesgo Bajo: hipoperfusión reversible o fija en un solo segmento ventricular. Riego Alto: hipoperfusión reversible o fija en dos o mas segmentos ventriculares, captación pulmonar de talio, o dilatación de la cavidad ventricular.

c) Criterios de riesgo del ecocardiograma con dobutamina

Riesgo Bajo: Asinergia en ${ }^{1-2}$ segmentos de distribución vascular coronaria. Función sistólica normal

Riesgo Moderado: Respuesta isquémica a altas dosis. Asinergia en mas de 2 segmentos de distribución vascular coronaria.

Riesgo Alto: Respuesta isquémica a bajas dosis. Asinergia en múltiples segmentos o en territorio de 203 vasos. Deterioro severo de la función sistólica.

3. Función ventricular: su evaluación es útil ya que existe una relación directa entre la disminución de la fracción de eyección (FE) y el aumento de la mortalidad. ${ }^{12}$ Se evalúa midiendo la FE con métodos no invasivos como el ecocardiograma y el ventriculograma radioisotópico con tecnecio. La FE normal es mayor al 50\%. Cuando disminuye a menos de $30 \%$, la mortalidad anual asciende al $40 \%$. La función ventricular puede deteriorarse luego de un infarto extenso, especialmente si es de la cara anterior o como consecuencia de isquemia crónica que produce una afección global del ventrículo izquierdo.

Bajo riesgo (RB) cuando la FE es mayor al 50\%, y la función sistólica es normal.

Riesgo moderado (RM) si la FE es de 40 a 50\%, (depresión leve a moderada de la función sistólica.)

Riesgo Alto (RA) si la FE es menor al $40 \%$ (compromiso severo de la función sistólica. )

Tabla 3. Categorias de riesgo 5

Teniendo en cuenta la extensión de la enfermedad, el grado de isquemia y la función ventricular, es posible clasificar a los pacientes con ACE en 4 categorías de riesgo:

\begin{tabular}{lccccc}
\hline & \multicolumn{2}{c}{ Bajo } & A & Moderado & Alto \\
& & I-II & III-IV & III-IV \\
\hline Clase funcional & I-II & II & + & + \\
\hline Infarto previo & - & $-/+$ & + & + \\
\hline FRC & $-1+$ & + & & \\
\hline Respuesta al & & & + & - \\
Tratamiento & + & + & RB RM & RA \\
\hline Función VI* & RB & RB RM & &
\end{tabular}

FRC factores de riesgo coronario

* Su evaluacion se reserva en general para los pacientes con riesgo clinico modera-

do B o Alto. La clase funcional se considera sin tratamiento antianginoso previo y

la respuesta al tratamiento es si mejora esta clase funcional.

\section{Recomendaciones de CCG para estratificación de riesgo 2}

1- Pacientes con riesgo alto por angina en CF III-IV. Recomendación Clase I, nivel de evidencia B.

2- Pacientes con riesgo moderado $\mathrm{B}$ y funcion ventricular de riesgo moderado. Recomendación Clase IIa, nivel de evidencia C.

\section{Conclusión}

Los médicos de atención primaria deben saber identificar adecuadamente a los pacientes con alto y bajo riesgo de infarto de miocardio y muerte súbita, para que aquellos con mayor riesgo puedan ser adecuadamente estudiados y tratados con procedimientos de revascularización (angioplastia y/ o cirugía), y aquellos con menor riesgo tratados médicamente en forma segura y efectiva (ver algoritmo final). En la próxima entrega nos dedicaremos al aspecto terapéutico de la ACE.

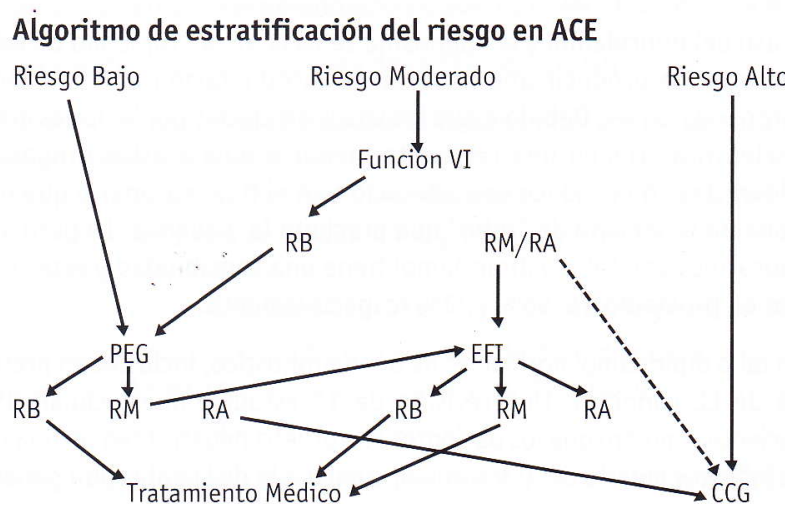

PG: Prueba Ergométrica EFI: Evaluación Funcional por Imágenes RB: Riesgo Bajo

RM: Riesgo Moderado RA: Riesgo Alto CCG: Cinecoronariografía

\section{Referencias}

1. Braunwald E; Heart Disease, a Textbook of Cardiovascular Medicine, 4 th. Edition 1992

2. ACC/AHA/ACP-ACIP Guidelines for the management of patients with chronic stable angina: Executive summary and reccomendations. Circulation, 1999;99:2829-48

3. Bertolassi ; Cardiología Clínica, 1999

4. Carbajal E, Prakash D. Coronary Heart Disease. Medical Clinics of North America 75 : 1063-1083, 1995

5. Sociedad Argentina de Cardiología, Asociación Argentina de Angiología y Cirugía Cardiovascular. Consenso de cardiopatía isquémica. Revista Argentina de Cardiología.

65, supl. II, 1997

6. Proudfit WL, Dolor de pecho, angina de pecho y estados relacionados. Heart Disease and Stroke $1993 ; 1: 9-14$

7. Diamond GA, Forester JS, Analysis of probability as an aid in the clincal diagnosis of coronary artery disease. N Engl J Med 1979; $300: 1350-1358$

8. Chaitman BR, Bourassa MG et al. Angiographic prevalence of high risk coronary artery disease in patients subsets (CASS). Circulation. 1981;64: 360-67

9. Chou T, Amidor T. Evaluating Coronary Aryery Disease Low Invasively; Which Test for whom ? WJM 1994; 161: 173-180

10. Mark D, et al. Prognostic value of treadmill exercise score in outpatients with suspected coronary artery disease. N. Engl. J. Med. $1991 ; 325$ :849-855

11. Tanimoto M, Pai RG, et al. Dobutamine stress echocardiography for the diagnosis and management of coronary disease. Clin Cardiol 1995; $18: 252-60$

12. A report of the American Heart Association/ American College of Cardiology, of the Cardiology Task Force (comitee on PTCA). Guidelinees for percutaneous transluminal coronary angioplasty. Circulation, $1993 ; 88,2987-300$

13. Yusuf S., et al. Effect of coronary by pass graft surgery on survival : overview of 10-year results from randomized trials. Lancet $1994 ; 344: 563-570$. 